\title{
Review: twin studies show that genes and individual environmental influences contribute to the aetiology of major depression
}

Sullivan PF Neale MC, Kendler KS. Genetic epidemiology of major depression: review and meta-analysis. Am J Psychiatry 2000 Oct;:157:1552-62.

\section{QUESTIONS: Is major depression (MD) familial? What are the relative contributions of genes and environment?}

\section{Data sources}

Studies were identified by searching Medline and reference lists of review articles.

\section{Study selection}

Studies were selected if an explicit distinction was made between unipolar MD and bipolar disorder, probands and their relatives were recruited systematically, diagnostic data were directly collected from all (or nearly all) participants, diagnostic criteria were operationalised, and assessors who made the diagnoses were blinded. Family studies had to include a comparison group. Twin studies also had to have zygosity determined by assessors who were blind to the co-twin's diagnosis.

\section{Data extraction}

Data were extracted on study design, country, source of participants, morbidity risk, and odds ratios. The results of family studies were pooled by using the MantelHaenszel method. For twin studies, structural equation modelling was used to estimate the variance associated with genetic influences, shared environmental influences, and environmental influences specific to the individual.

\section{Main results}

5 family studies ( 4 from the US and 1 from Germany) met the selection criteria. Participants consisted of medical patients ( 1 study), surgical patients (1 study), and the general population (3 studies). An association existed between $\mathrm{MD}$ in the proband and $\mathrm{MD}$ in first degree relatives (pooled odds ratio 2.8, 95\% CI 2.3 to 3.5). No adoption studies met the selection criteria. 6 twin studies (2 from the US, 2 from Sweden, 1 from the UK, and 1 from Australia) of $>21000$ participants met the selection criteria. 4 studies had community samples, and 2 studies had clinical samples. The best fitting model showed that the variance in liability to MD was accounted for by additive genetic effects (point estimate $37 \%$, CI $33 \%$ to $42 \%$ ) and individual specific environmental effects (point estimate $63 \%$, CI $58 \%$ to $67 \%$ ). The estimate of the variance from environmental influences specific to an individual included measurement error and gene environment interactions. The contribution of shared environmental influences was minimal (point estimate $0 \%$, CI $0 \%$ to $5 \%$ ).

For correspondence: Dr P F Sullivan,

Department of Psychiatry, Virginia Institute for Psychiatric and Behavioral Genetics, $P O$ Box 980126, Richmond, VA 23298-0126, USA

$\mathrm{Fax}+1804828$ 1471 .

\section{Conclusions}

First degree relatives of people with major depression (MD) have an increased risk of MD. The variance in liability to $\mathrm{MD}$ is accounted for by additive genetic effects and environmental effects specific to an individual.

\section{COMMENTARY}

Despite the substantial health problems caused by unipolar depression or MD, family, twin, and adoption studies that examine unipolar illness are fewer than those that examine bipolar disorder or schizophrenia. Consequently, this metaanalysis by Sullivan $e t$ al of twin and family studies of MD is important. The pooled data from rigorously chosen studies indicate that genes contribute about one third and individual environmental factors about two thirds of the variance in liability to develop MD. These findings are surprisingly consistent across the different studies. The meta-analysis also confirms that the genetic risk factors are less prominent for unipolar than for bipolar disorder, where nearly $80 \%$ variance in liability is due to genetic factors with proportionately less variance from individual environmental factors. ${ }^{1}$

Clinicians are well aware that depression appears to run in some families. What is probably less well known is that this familiality is mainly due to shared genes rather than shared family experiences. In Sullivan et al's review, shared environmental factors provided only a negligible contribution to the variance. This is also a consistent finding in studies of adults, although for depressive symptoms in early childhood, shared or family environment provides a much greater contribution to the variance. ${ }^{2}$ The influence of family environment diminishes in adolescence.

What the review's methodology also cannot tell us is which specific genes or environmental factors are influential, although there are clues to both. The mode of inheritance in unipolar depression is not Mendelian, and is most probably polygenic. This means that several genes (maybe even dozens) act together additively to produce a susceptibility to develop depression when other risk factors are also present. The mode of action of the main groups of antidepressants on the genetically determined serotinergic and noradrenergic neurotransmitter systems could provide a starting point for molecular genetic studies of MD.

In addition, the most well recognised environmental risk factor is the relation between certain types of severe, threatening life events to the onset of depressive episodes. Indeed, depression is unique among psychiatric syndromes in that a large body of research has clearly shown the relation between life events, as environmental risk factors, and episode onset. There are established and well designed methodologies for measuring life events. ${ }^{3}$ Consequently, the next challenge is the integration of genetic and life event research to explore further the relation between genes and environment in MD.

Anne Farmer, MD, FRCPsych Institute of Psychiatry, King's College London London, UK

1 Farmer AE. The genetics of depressive disorders. International Review of Psychiatry 1996;8:369-72.

2 Thapar A, McGuffin P. A twin study of depressive symptoms in childhood. Br J Psychiatry 1994;165:259-65.

3 Brown GW, Harris TO. Social origins of depression: a study of psychiatric disorder in women. London: Tavistock Publications, 1978. 